\title{
Malignant melanoma of prepuce: case report
}

\author{
J A FORRER AND D L SUGRUE
}

From the Department of Genitourinary Medicine, North Staffordshire Hospital Centre, Stoke-on-Trent, Staffordshire

SUMMARY A 58 year old man presented with what seemed to be a simple penile lesion, but which on subsequent histological examination proved to be a malignant melanoma. The lesion was excised by circumcision. No further treatment was considered to be necessary.

\section{Introduction}

In departments of genitourinary medicine, lesions affecting the genitalia that are not sexually transmitted are seen increasingly. ${ }^{1}$ It is important therefore to remember that patients with malignant disease may initially present to a genitourinary clinic. We show this with a case of malignant melanoma of the penis, an extremely rare form of neoplasm.

\section{Case report}

A 58 year old man presented at the department of genitourinary medicine at this hospital in November 1984 with a painless growth of eight months' duration on the end of his penis. The fact that it began to exude fluid from its surface and had bled on one occasion caused the patient to seek medical advice. He had no history of sexually transmitted disease, and there were no urinary symptoms.

On examination a pedunculated non-pigmented lesion $2.0 \times 1.4 \times 0.7 \mathrm{~cm}$ arose from the right side of the end of the prepuce. The surface of the lesion was smooth and moist. There was no palpable enlargement of the inguinal lymph nodes. Serological tests for syphilis gave negative results. Routine urine analysis gave normal results, and a two glass urine test showed no evidence of anterior urethritis.

The patient was referred for surgical excision of the lesion to assess its exact nature. In December 1984 he was admitted to hospital. Routine investigations on admission including full blood count, urea and electrolyte concentrations, chest radiography, and midstream urine tests gave normal results. The lesion

Address for reprints: Dr J A Forrer, Department of Genitourinary Medicine, Central Outpatients Department, North Staffordshire Hospital Centre, Hartshill Road, Stoke-on-Trent, Staffordshire ST4 7PA

Accepted for publication 5 March 1986 was removed by circumcision, postoperative recovery was uneventful, and the patient was discharged home the following day.

Histopathological findings (figure) were those of an ulcerated spindle cell malignant melanoma $0.65 \mathrm{~cm}$ deep. Excision seemed to be complete.

\section{Discussion}

Malignant melanomas constitute $1.3 \%$ of all malignancies. Of all malignant melanomata, those of the genitourinary tract acount for less than $1 \%{ }^{2}$

Between $65 \%$ and $85 \%$ of malignant melanomata arise from pre-existing naevi and $20 \%$ arise de novo. In about $4 \%$ of cases there is evidence of metastatic spread but the primary melanoma cannot be localised, which suggests that these lesions have undergone spontaneous regression. Of the melanomas arising in pre-existing naevi, most occur on anatomical areas subjected to trauma, such as the palms, soles, and external genitalia.

Malignant melanoma of the penis is rare. Since the first reported case in $1882,{ }^{3} 56$ cases have been mentioned in published reports. It is found most commonly in patients aged between 40 and 70 , and the most common site is the glans (in two thirds of cases). Its appearance to the naked eye may be that of a papule, ulcer, or protuberant mass. Often the lesion has been present for years, and the onset of increasing pigmentation, ulceration and bleeding, or a rapid increase in size leads to its diagnosis. Diagnosis may be delayed because the patient may be reluctant to seek medical advice (through fear of the possible malignancy of the lesion) or because the lesion may be misdiagnosed as inflammatory or infectious. ${ }^{4}$ Microscopical appearances are identical to those of melanomata arising elsewhere on the skin.

Spread is by local infiltration into the corpora cavernosa and via the lymphatics to the superficial inguinal nodes. Spread to deep pelvic nodes, or via the 


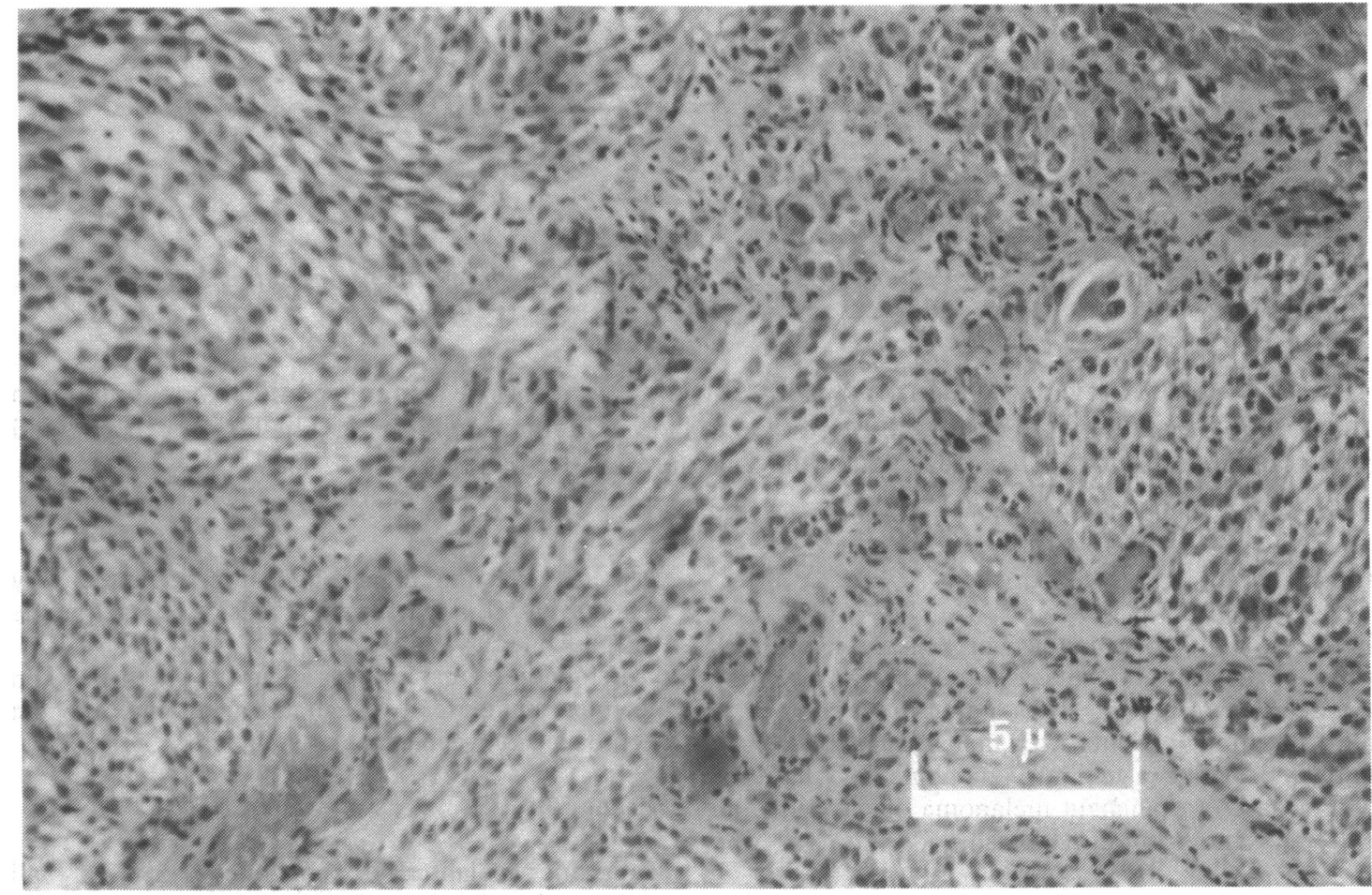

FIGURE Section of penile lesion showing melanocytes with malignant features. (Haematoxylin and eosin.)

bloodstream to distant sites, occurs later. Clinical stages are: (1) localised disease not affecting regional lymph nodes, (2) tumour found in regional lymph nodes, and (3) tumour found in other lymph nodes, or distant metastases. Unfortunately, clinical examination is not always a reliable indication of whether or not the inguinal nodes are affected.

The prognosis is generally poor. Metastatic disease is already present at the time of diagnosis in $50 \%$ of patients. Overall, only $15 \%$ of patients survive five years.

Surgery is the mainstay of treatment for penile melanoma; results of treatment are difficult to evaluate because numbers of patients are very small. For lesions of the prepuce, the recommended treatment is partial amputation with bilateral superficial inguinal node dissection. ${ }^{5}$ If deep pelvic nodes are affected their dissection is unlikely to result in operative cure, as the disease is likely to be widely spread.

In one series of 14 patients with stage I melanoma treated by partial amputation with or without inguinal node dissection or radiotherapy, not one patient survived longer than two years. ${ }^{6}$ An appreciable risk of recurrence in the penile stump after partial amputation led to the suggestion that, for lesions of the glans, total amputation with block dissection of inguinal nodes would be more justified. Of eight patients who were treated by total amputation, four were alive after five years.

Radiotherapy is not considered to be of any value in the treatment of the primary lesion because melanoma is generally considered to be resistant.

Chemotherapy with dacarbazine (DTIC) has been found to be effective in some cases of cutaneous malignant melanoma, with response rates of $23-25 \%$. It has not, however, been used in patients with penile melanoma. Adjunctive chemotherapy has been used in some cases. Ellis and White describe a case in which amputation was followed by endolymphatic treatment using radioactive lipiodol. Twenty one months after operation the patient was well and clinically free from metastases. ${ }^{7}$

In our patient the lesion was on the prepuce, and local excision in the form of circumcision was carried out. On receipt of the histopathology report it was decided not to carry out a prophylactic block dissection of the inguinal nodes but to adopt a wait and see policy. We thought that subjecting the patient to a more mutilating surgical procedure was not justified, bearing in mind the generally poor prognosis for this type of neoplasm. He was followed up at intervals of three months and at the most recent visit, 14 months after excision, he remained symptomless and had no clinical evidence of recurrence. 
We thank Mr P M Higgins, Consultant Urologist, North Staffordshire Hospital Centre, for his help with this report.

\section{References}

1. McMillan A. Genitourinary Medicine. British Journal of Venereal Diseases 1984;60:213.

2. Stein BS, Kendall AR. Malignant melanoma of genitourinary tract. J Urol 1984;132:859-68.
3. Gross SD. A system of surgery: pathological, diagnostic, therapeutic, and operative. 6th ed. Philadelphia: WB Saunders, 1882; vol 2:834.

4. Begun FP, Grossman HB, Diokno AC, Sogani PC. Malignant melanoma of the penis and male urethra. J Urol 1984;132:123-5.

5. Bracken RB, Diokno AC. Melanoma of the penis and the urethra, two case reports and review of the literature. J Urol 1974;111:198-200.

6. Khezri AA, Dounis A, Roberts JBM. Primary malignant melanoma of the penis. Two cases and a review of the literature. Br J Urol 1979;51:147-50.

7. Ellis $\mathbf{M}$, White WF. Malignant melanoma of penis: endolymphatic therapy with 131-I lipiodol. $B r J$ Surg 1968;55:238-41. 